\title{
Dynamic retraction: a novel technique for robot-assisted simple prostatectomy
}

\author{
Syed Johar Raza, Allison Marie May, Shanoon Theresa Moore, Zachary Adam Hamilton \\ Division of Urology, Department of Surgery, Saint Louis University Hospital, MO, USA \\ Correspondence to: Syed Johar Raza, MD. Division of Urology, Department of Surgery, Saint Louis University Hospital, 3635 Vista Ave, 3rd Floor \\ Desloge Towers, St. Louis, MO 63110, USA. Email: johar.syed@health.slu.edu.
}

\begin{abstract}
Open simple prostatectomy is the mainstay treatment for obstructive urinary symptoms due to benign prostate enlargement, failing medical and/or transurethral resection therapies. Due to widespread utilization of robot-assisted techniques for radical prostatectomy, robot-assisted simple prostatectomy (RASP) has gained similar popularity. Availability of dual console on newer robotic platforms has improved the ability to engage the 4th arm, providing dynamic use during the operation. In this case report we describe the use of dynamic retraction for a 59-year-old male who underwent RASP.
\end{abstract}

Keywords: Robotic surgical procedures; prostate; urologic surgical procedures

Received: 04 July 2018; Accepted: 30 July 2018; Published: 02 August 2018.

doi: 10.21037 /jovs.2018.07.07

View this article at: http://dx.doi.org/10.21037/jovs.2018.07.07

\section{Introduction}

Bladder outflow obstruction from benign prostatic enlargement is one of the most common urologic ailments affecting middle age men. Various treatment modalities are available, starting from medical management to transurethral procedures or more invasive removal of the prostatic adenoma (1). Patients, who continue to remain symptomatic despite adequate medical or transurethral treatments, or those with significantly enlarged prostatic adenoma, are candidates for more invasive surgery. Traditionally this has been performed as open simple prostatectomy, involving trans-vesical enucleation of the prostatic adenoma. This procedure, despite being the most definitive way to treat prostatic enlargement, is associated with significant peri- and post-operative morbidity (2). The use of minimally invasive approach with regards to radical prostatectomy has encouraged the utilization of laparoscopic or robot-assisted simple prostatectomy (RASP) with proven benefits (3).

The robot-assisted approach is employed in a manner, similar to robot-assisted radical prostatectomy. At baseline the robot offers 4 arm approach with 3 working arms and 1 camera arm. The 3 rd working arm or commonly called 4th arm is used by alternating with one of the other working arms, rendering it deactivated while the other 2 arms are in use. Newer robotic platforms with dual console function allow the nonoperating surgeon to retain the control of the 4th arm, leaving it activated and thus allowing simultaneous use of all 3 working arms. The activated 4th arm can then be put to a continuous use during the procedure. This spares the operating surgeon the process of activating, repositioning and then deactivating the 4th arm for retraction, thus providing dynamic retraction. Because the presence of a large prostatic adenoma can obstruct the surgical plane, the use of dynamic retraction can be helpful for RASP. In this case report we demonstrate the use of dynamic retraction during RASP, allowing the senior surgeon to provide dynamic retraction and guidance, while the junior surgeon performs the procedure.

\section{Case presentation}

A 59-year-old male presented to the Urology clinic for further management of recurrent hematuria, lower urinary tract symptoms, and urinary retention. His past medical history was insignificant but had a strong family history of prostate cancer. He previously underwent a negative 

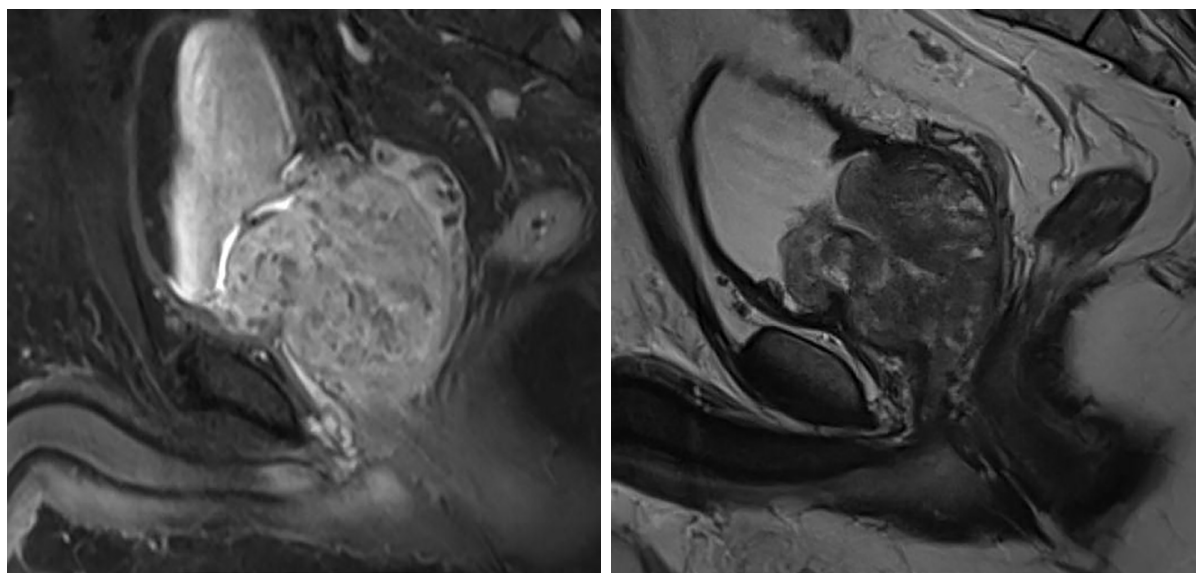

Figure 1 Sagittal views of the magnetic resonance imaging of the pelvis demonstrating a very large prostate gland.

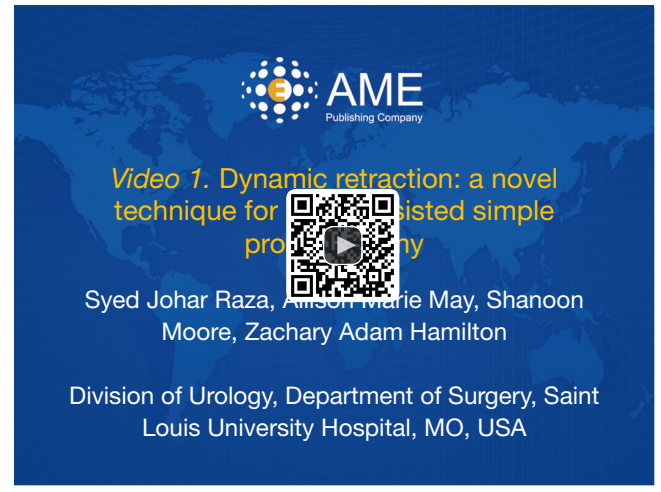

Figure 2 Dynamic retraction: a novel technique for robot-assisted simple prostatectomy (4).

Available online: http://www.asvide.com/article/view/26248

prostate biopsy for elevated PSA and also a negative magnetic resonance imaging (MRI) guided biopsy. Although the biopsy was negative for carcinoma, his MRI revealed a significantly enlarged prostate despite two previous treatments of transurethral resection of the prostate (Figure 1). As the patient continued to be symptomatic with hematuria and urinary retention, he elected to proceed with RASP.

Patient preparation and positioning was performed similar to a radical prostatectomy approach, with the supine position and steep Trendelenburg. Pneumoperitoneum was established using the Veress needle and standard port placement was done. The camera port was placed $2 \mathrm{~cm}$ cephalad to the umbilicus. Two robotic arms were placed on the patient's left, 8 and $16 \mathrm{~cm}$ lateral to the umbilicus. One robotic arm was placed on the patient's right, $8 \mathrm{~cm}$ lateral to the umbilicus, and an assistant port was placed on the patient's right, $16 \mathrm{~cm}$ lateral to the umbilicus. The robot was docked. The junior surgeon was transferred the controls of the camera, 1st, and 3rd working arms. The senior surgeon retained the control of the 4th arm and provided dynamic retraction as needed (Figure 2). Space of Retzius was exposed and the bladder neck was identified. A transverse cystostomy was performed $2 \mathrm{~cm}$ proximal to the perceived level of the bladder neck. The balloon of the Foley catheter was deflated and catheter pulled back. The large median lobe of the prostate was identified. A size 0 silk suture was placed through the prostate tissue and the suture pulled away to expose the incision line to enucleate the adenoma. The dynamic retraction of the median lobe provided uninterrupted and continuous excision of the adenoma. This allowed the senior surgeon to retract the prostate adenoma, guide the junior surgeon during excision, and allowed for the bedside assistant to focus on suction to keep a bloodless field. To assist the anterior dissection, we placed 2 silk sutures at the anterior bladder wall and extracted the sutures through the skin just above the pubic symphysis, allowing for percutaneous traction by the bedside assistant. This allowed exposing the anterior plane of excision. Once the adenoma was mobilized off the surrounding pseudo capsule, the urethra within the capsule was incised and adenoma removed. The posterior urethral plate was reconstructed with continuous 2-0 V-Lock suture, and a final Foley placed. The cystostomy was closed in 2 layers. The estimated blood loss was $400 \mathrm{~mL}$ with robot dock time of $200 \mathrm{~min}$. Patient remained in house for 23 hours, without the need for bladder irrigation, and was discharged home without complications. A post cystogram 


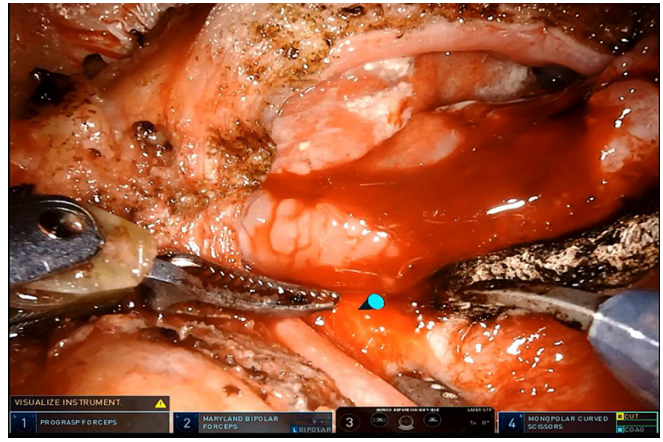

Figure 3 Operative image showing the senior surgeon's nonoperative right hand used as pointer to identify the site if incision.

voiding trial was successful. At 3-month follow-up in clinic, patient was asymptomatic, with a prostate specific antigen of 0.2 .

\section{Discussion}

Open simple prostatectomy has long been considered a valuable and effective option for treatment of very large prostatic adenoma. However, the procedure is reported to be associated with a $13-37 \%$ complication rate (2). The increasing use of laparoscopic and robotic surgery has attempted to mitigate the complications of simple prostatectomy. Sotelo et al. reported the first RASP in 2008 (5). Since then, the robotic approach has become a popular choice for this procedure. Autorino et al. reported the largest multi-institutional series of 487 patients who underwent RASP (6). He reported a mean operative time of $145 \mathrm{~min}$, with mean blood loss of $200 \mathrm{~mL}$. Mean hospital stay was 2 days, with a $1 \%$ peri-operative transfusion rate. Studies have also reported outcomes comparable to open and laparoscopic techniques. Using a propensity match analysis Sorokin et al. compared 59 matched cases of open prostatectomy and RASP (3). They noted significantly decreased blood loss, hematocrit drop and rate of blood transfusion, favoring the robotic approach. Overall complications were comparable in both groups with similar functional outcome; however, the robotic approach resulted in significantly shorter hospital stay. Interestingly all studies have reported an increased operative time for robotic approach compared to the open. This could be related to additional time spent in establishing pneumoperitoneum and port placement, robot docking and undocking, specimen extraction and dependence of the console surgeon on bed side assistance. Surgical flow disruptions are known to cause increased operative time, most commonly noted at the time of robot docking and dependent on surgeon's experience (7). Additionally, training cases are also known to increase operative times and flow disruptions.

In this case, we used the novel technique of dynamic retraction for additional assistance during RASP. To our knowledge this is the first time this method of assistance has been reported. The advantages of this technique are not limited to simple prostatectomy, but can be utilized for any robot-assisted case, with dual console availability. Use of dual console has previously been associated with improved operative outcomes. Morgan et al. compared the operative outcomes of single $v s$. dual console use during robot-assisted radical prostatectomy (8). They reported that use of dual console resulted in shorter operative time with fewer intra- and post-operative complications. In addition higher grade complications were reported with single console use. Dynamic retraction would thus be anticipated to improve the operative outcomes in training cases. The ability to manipulate tissue within the surgical working space and provision of optimal exposure is the intended benefit of this technique. It allows for dual operative surgeon capability during robotic procedures, in addition to a bedside assistant. This effect is particularly helpful for simple prostatectomy cases, where a large median lobe is obstructing the enucleation plane. Furthermore, while the senior surgeon is dynamically retracting with the 4th arm, the other non-functional hand can continue to use the on-screen cursor for surgical direction. This helps in identifying important structures and pinpointing the area of incision/dissection (Figure 3). Despite such advantageous use, there are some limitations to the use of this dynamic retraction. Firstly, this feature is only available with the dual console, hence associated with additional cost of the second console. Secondly, in order to move the 4th arm, the instrument must be maintained in the field of vision. This may restrict the primary surgeon to a 'zoomed out' view in order to attain maximal benefit out of dynamic retraction.

The use of novel dynamic retraction technique appears to be a simple and effective way to assist during robot-assisted surgeries. Based on this initial experience, we encourage all robotic surgeons to utilize this technique, especially in training cases to help accelerate the learning process for junior surgeons. In the future, it will be interesting to translate this technique to a larger cohort with comparative analysis and determine if dynamic retraction can positively affect various operative parameters during robot-assisted surgeries. 


\section{Acknowledgments}

Funding: None.

\section{Footnote}

Conflicts of Interest: All authors have completed the ICMJE uniform disclosure form (available at http://dx.doi. org/10.21037/jovs.2018.07.07). The authors have no conflicts of interest to declare.

Ethical Statement: The authors are accountable for all aspects of the work in ensuring that questions related to the accuracy or integrity of any part of the work are appropriately investigated and resolved. All procedures performed in studies involving human participants were in accordance with the Helsinki Declaration (as revised in 2013). Written informed consent was obtained from the patient for publication of this Case Report and any accompanying images. A copy of the written consent is available for review by the Editor-in-Chief of this journal.

Open Access Statement: This is an Open Access article distributed in accordance with the Creative Commons Attribution-NonCommercial-NoDerivs 4.0 International License (CC BY-NC-ND 4.0), which permits the noncommercial replication and distribution of the article with the strict proviso that no changes or edits are made and the original work is properly cited (including links to both the formal publication through the relevant DOI and the license). See: https://creativecommons.org/licenses/by-nc-nd/4.0/.

doi: 10.21037/jovs.2018.07.07

Cite this article as: Raza SJ, May AM, Moore ST, Hamilton

ZA. Dynamic retraction: a novel technique for robot-assisted simple prostatectomy. J Vis Surg 2018;4:160.

\section{References}

1. American Urological Association Guideline: management of benign prostatic hyperplasia (BPH). 2018. Available online: http://www.auanet.org/guidelines/benign-prostatichyperplasia/lower-urinary-tract-symptoms-(2018)

2. Ferretti M, Phillips J. Prostatectomy for benign prostate disease: open, laparoscopic and robotic techniques. Can J Urol 2015;22:60-6.

3. Sorokin I, Sundaram V, Singla N, et al. Robot-Assisted Versus Open Simple Prostatectomy for Benign Prostatic Hyperplasia in Large Glands: A Propensity Score-Matched Comparison of Perioperative and Short-Term Outcomes. J Endourol 2017;31:1164-9.

4. Raza SJ, May AM, Moore S, et al. Dynamic retraction: a novel technique for robot-assisted simple prostatectomy. Asvide 2018;5:661. Available online: http://www.asvide. com/article/view/26248

5. Sotelo R, Clavijo R, Carmona O, et al. Robotic simple prostatectomy. J Urol 2008;179:513-5.

6. Autorino R, Zargar H, Mariano MB, et al. Perioperative Outcomes of Robotic and Laparoscopic Simple Prostatectomy: A European-American Multi-institutional Analysis. Eur Urol 2015;68:86-94.

7. Dru CJ, Anger JT, Souders CP, et al. Surgical flow disruptions during robotic-assisted radical prostatectomy. Can J Urol 2017;24:8814-21.

8. Morgan MS, Shakir NA, Garcia-Gil M, et al. Singleversus dual-console robot-assisted radical prostatectomy: impact on intraoperative and postoperative outcomes in a teaching institution. World J Urol 2015;33:781-6. 